\title{
Design of Split Transmitter/Receiver Installation on Roadside Unit for Infrared-based Parking System
}

\author{
Hsin-Chuan Chen, ${ }^{1}$ Rong-San Lin, ${ }^{2 *}$ Chiou-Jye Huang, ${ }^{3}$ and Chang-Hsiang Tsao ${ }^{4}$ \\ ${ }^{1}$ School of Computer Engineering, University of Electronic Science and Technology of China, \\ Zhongshan Institute, Zhongshan 528402, China \\ ${ }^{2}$ Department of Computer Science and Information Engineering, \\ Southern Taiwan University of Science and Technology, Tainan City 710, Taiwan \\ ${ }^{3}$ School of Electrical Engineering and Automation, Jiangxi University of Science and Technology, \\ Ganzhou 341000, China \\ ${ }^{4}$ Department of Electronic Engineering, St. John's University, New Taipei City 25135, Taiwan
}

(Received November 20, 2019; accepted June 9, 2020)

Keywords: infrared signal, split TX/RX installation, sunlight interference, signal interference

More parking systems are developing toward automatic identification (ID) and control, where infrared (IR) technology is a low-cost and the most used approach for data transmission and ID. However, interference is caused by sunlight or two neighboring vehicles in a conventional IR-sensor-based parking system; therefore, a new methodology using a split transmitter/receiver (TX/RX) installation on a roadside unit (RSU) is proposed in this paper. The TX and RX modules are placed upward and downward on an on-board unit (OBU) device, respectively. Therefore, the proposed parking system can reduce the impact of sunlight on the RX module of the OBU device and reduce the signal interference caused by two neighboring vehicles in the sensing zone. Furthermore, the ID stability of the conventional RSU system can be increased.

\section{Introduction}

When a vehicle approaches the entrance/exit of a parking lot, the driver uses a wireless remote controller or radio frequency identification (RFID) card $^{(1)}$ to open the parking gate. Although these two identification (ID) approaches are simple and cheap, it is inconvenient for the driver because manual operation is required. Using the technology of license plate recognition realizes automatic ID and control, ${ }^{(2,3)}$ however, such a parking system requires expensive infrastructure and the ID rate is not $100 \%$ because image recognition is easily affected by many environmental factors, such as light conditions and the angle of imaging. ${ }^{(2)}$ An uncooled infrared (IR) camera, ${ }^{(4)}$ a type of thermal sensor for measuring IR radiation, ${ }^{(5)}$ is also used to capture nighttime vehicle images, and it can reduce image noise when stabilized at an operating temperature. There are some image processing technologies that can be used to enhance the performance of parking systems using license plate recognition. For example, a panoramic image stitching system ${ }^{(6)}$ has been applied to the automobile rear view, and $360^{\circ}$

*Corresponding author: e-mail: rslin@stust.edu.tw https://doi.org/10.18494/SAM.2020.2716 
images based on the Haar wavelet transform were acquired through multiple monitors to reduce car accidents. In addition, vision-based nighttime vehicle detection ${ }^{(7,8)}$ can be used to determine the number of lane-straddling vehicles by identifying lane lines and recognizing vehicle lights, ${ }^{(7)}$ or to effectively detect vehicles at night by using a fog lamp elimination method and adaptive vehicle mask training. ${ }^{(8)}$ For the Electronic Toll Collection (ETC) system of freeways using E-tag ID, ${ }^{(9,10)}$ no on-board unit (OBU) device needs to be set in the vehicle, and it can automatically complete ID only by pasting a passive E-tag on the vehicle. However, in such a system, especially for a long communication distance, people may worry about the RF radiation harming human health if an RF station with high power is established near homes or a community. ${ }^{(11)}$ Moreover, the RF band used by the ETC system partially overlaps with that presently used for mobile communication, which may cause interference when using mobile phones, and the system cost is still high.

IR communication, a type of wireless communication technology, uses an IR signal to transfer data between transmitters and receivers. Owing to its low implementation cost, it is widely applied to remote controls for many electrical appliances. ${ }^{(12)}$ However, sunlight or fluorescent light easily disturbs IR communication if the transmitter directly sends data by an IR signal with no further processes, and thus the IR receiver cannot correctly read the data. Therefore, data encoding/decoding and modulation/demodulation are necessary for IR communication. ${ }^{(13)}$ An IR signal has a characteristic of direction, which means that an IR LED obtains the highest radiation intensity when its emitting angle is $0^{\circ}$, but the radiation intensity decreases away from $0^{\circ}{ }^{(14)}$ In summary, IR technology is suitable for slow and short communication in a space with few obstacles. ${ }^{(15)}$ In contrast to RF radiation, an IR signal causes almost no damage to the human body in most cases. Because of the low implementation cost, safety, and easy operation of IR communication, a parking system using IR communication is suitable for small and medium-size parking lots. However, interference is caused by sunlight or two neighboring vehicles in a conventional IR-sensor-based parking system; therefore, in this paper, we propose a new methodology using a split transmitter/receiver (TX/RX) installation on a roadside unit (RSU) to solve these problems.

This paper is organized as follows. Section 2 reviews the basic structure and operation of the conventional IR-based parking system, Sect. 3 introduces the proposed method of installing transmitters and receivers and how to solve the interference problems, Sect. 4 gives experimental results and analysis, and Sect. 5 concludes the paper.

\section{Conventional IR-based Parking System}

The basic structure of a parking system based on IR communication is shown in Fig. 1, ${ }^{(11,13)}$ which includes an OBU device set in the vehicle and an RSU system ${ }^{(15,16)}$ installed at the entrance/exit of a parking lot. Both the OBU device and the RSU system require a pair of TX/RX modules to transmit or receive the IR signal to/from each other to create a down/up link, where the TX/RX modules of the OBU device face upward and the TX/RX modules of the RSU system face downward. The operation of the conventional IR-based parking system is described as follows: $:^{(11,13,15,16)}$ 


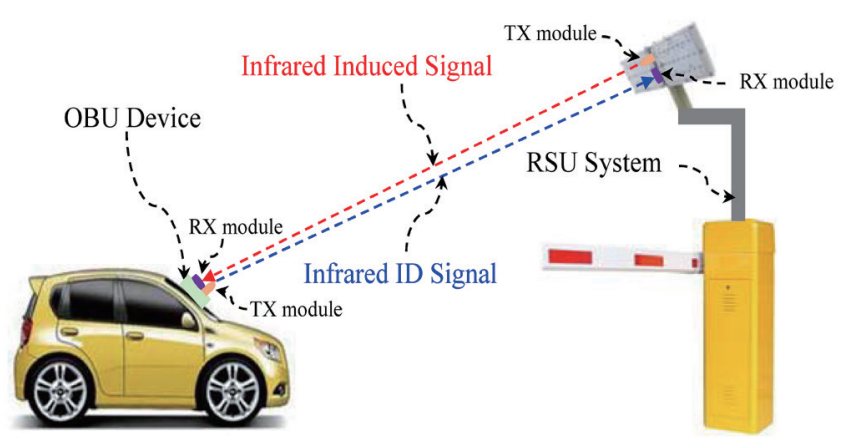

Fig. 1. (Color online) Parking system using IR communication.

(1) When the car enters the sensing zone, the RSU system sends an induced signal.

(2) Once the OBU device receives this signal, it transmits an ID signal indicating its ID code back to the RSU system.

(3) After recognition, the RSU system opens the parking gate if the ID code is correct.

\subsection{Sunlight interference}

In the conventional parking lot based on IR ID, the IR TX and RX modules of the RSU system are placed together on a high frame, and transmit and receive signals downward at an oblique angle. Therefore, the OBU device of the vehicle must be installed on the car windshield to send and receive IR signals from above. Sunlight, which is part of the electromagnetic radiation emitted by the sun, is a mixture of electromagnetic waves ranging from IR to ultraviolet (UV) rays. ${ }^{(17)}$ Therefore, the operation of the IR receiver is strongly affected by sunlight; although there is less impact on the downward receiver of the RSU, the upward receiver of the OBU device remains almost saturated when exposed to sunlight, meaning that it cannot receive the IR-induced signal from the RSU system. Naturally, the TX module of the OBU device does not send an IR ID signal back to the RSU system at this moment, so the RSU system cannot correctly identify the vehicle. Figure 2 shows how the RX module of the OBU device is affected by sunlight.

\subsection{Signal interference between two neighboring vehicles}

Since the conventional RSU system uses a top-down transmitting mode, the IR signal emitted by the RSU's transmitter will cover a limited range in which vehicles can detect this induced signal, which is called the sensing zone. The distance of the emission depends on the energy intensity of the transmitter at a given current. Furthermore, its effective sensing distance is also related to the setup height $h_{T}$ and downward emitting angle $\theta$ of the IR transmitter. ${ }^{(15,16)}$ Figure 3 shows the relationship between $d_{S}$ and $h_{T}$; the effective sensing distance $d_{S}$ is

$$
d_{S}=h_{T} \times \tan \theta .
$$




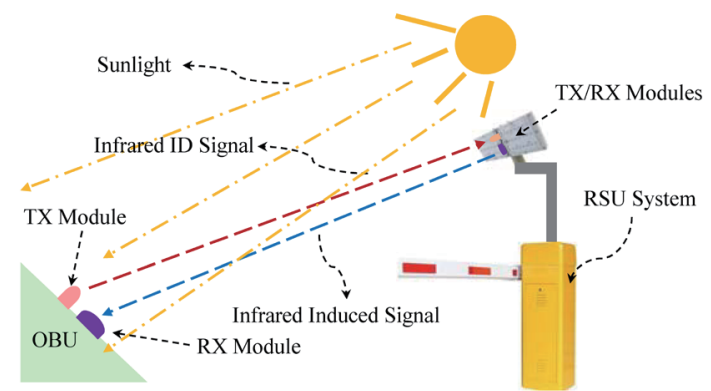

Fig. 2. (Color online) Impact of sunlight interference on RX module of OBU device.

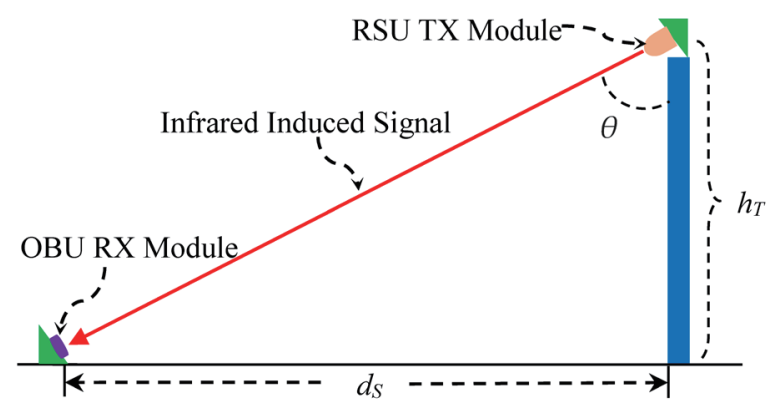

Fig. 3. (Color online) Relationship between setup height and downward emitting angle of TX module.

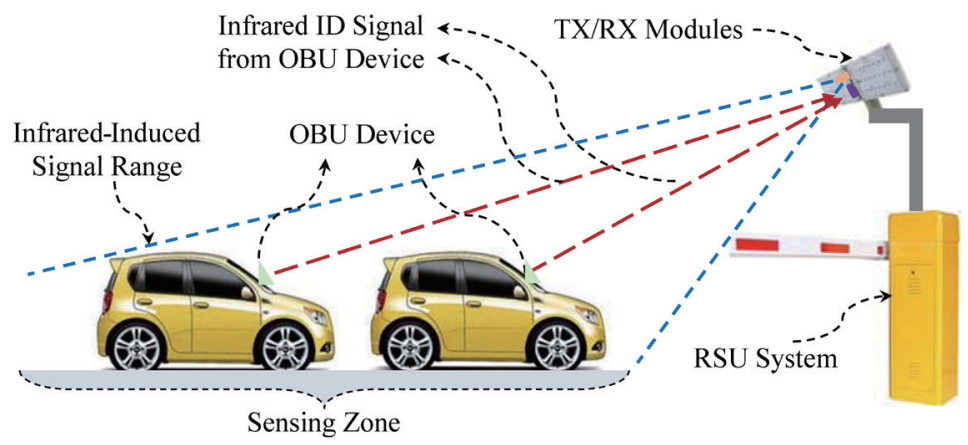

Fig. 4. (Color online) Signal interference for two neighboring vehicles in sensing zone.

When two vehicles enter the sensing zone at the same time, their OBU devices concurrently receive the induced signal from the RSU system and then return their ID signals back to the RSU system. These two signals will interfere with each other in air, ${ }^{(18)}$ as shown in Fig. 4, which will distort the ID codes from the vehicles in the sensing zone, and thus the RSU system cannot recognize the correct ID signal. This often happens when vehicles are waiting in line to enter or leave a parking lot, and thus the RSU system must use a camera to capture photos of these vehicles for further processing.

\section{Proposed Split TX/RX Installation}

To solve the problem of interference caused by sunlight or two neighboring vehicles in the conventional IR-based parking system, in this paper, we propose the method of split installation of the transmitter and receiver modules on the RSU. In the proposed IR-based parking system, the TX and RX modules on the OBU device are placed upward and downward, respectively, where the OBU device is mounted below the windshield of the vehicle. In the RSU system, the TX module is placed upward at the bottom of the RSU equipment, and the downward RX module is set at the top of the RSU equipment. Similar to the concept of conventional TX/RX installation, the sensing distance $d_{S}$ of the proposed split TX/RX installation shown in Fig. 5 is given by 


$$
d_{S}=h_{R} \times \cot \theta
$$

where $h_{R}$ is the height of the OBU device in the vehicle and $\theta$ is the upward emitting angle of the IR transmitter on the RSU equipment. The following subsections describe the design of the TX/RX modules and discuss how to reduce sunlight interference and the signal interference between two neighboring vehicles.

\subsection{Design of TX/RX modules}

The TX and RX modules of the OBU device and RSU equipment are almost the same but have different assigned encoding addresses. Figure 6 shows the architecture of the TX/RX modules, ${ }^{(11)}$ where the controller is used to handle the address encoding/decoding, read data from the decoder, and activate the IR transmitter. The RX module includes an IR receiver with demodulation and a data decoder, where the IR receiver is typically a standard IR receiver module, which is used to receive the IR signal and remove its carrier of $38 \mathrm{kHz}$ frequency, and then outputs the demodulated signal. This module integrates a p-intrinsic-n (PIN) diode, a preamplifier, an automatic gain control (AGC) circuit, a band pass filter, and an output stage into a package, and it only provides three pins for convenient usage. ${ }^{(19)}$ The TX module is composed of a data encoder, a modulator of the $38 \mathrm{kHz}$ carrier, and an IR transmitter. The IR transmitter consists of several IR LEDs and a driving transistor, and the IR intensity depends on the number of IR LEDs and the current flowing through them. ${ }^{(11)}$

\subsection{Sunlight interference reduction}

In our proposed system, the TX modules of the RSU equipment and OBU device face upward to transmit the IR-induced and IR ID signals, respectively. Although sunlight may directly shine on these two TX modules, it will not affect their transmission of IR signals. The RX modules of the OBU device and RSU equipment face downward to receive the IR-induced and IR ID signals, respectively. Therefore, they can avoid direct exposure to sunlight, as shown in Fig. 7, which will reduce the impact of sunlight interference.

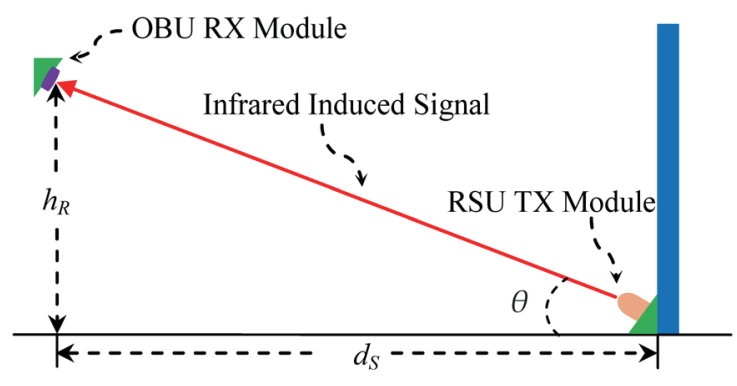

Fig. 5. (Color online) Sensing distance of proposed installation method.

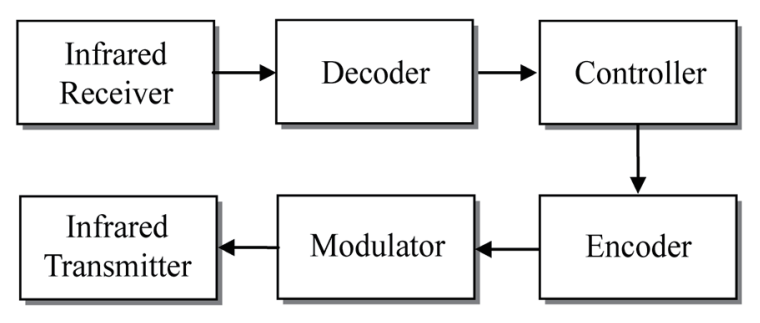

Fig. 6. Architecture of TX/RX modules. 


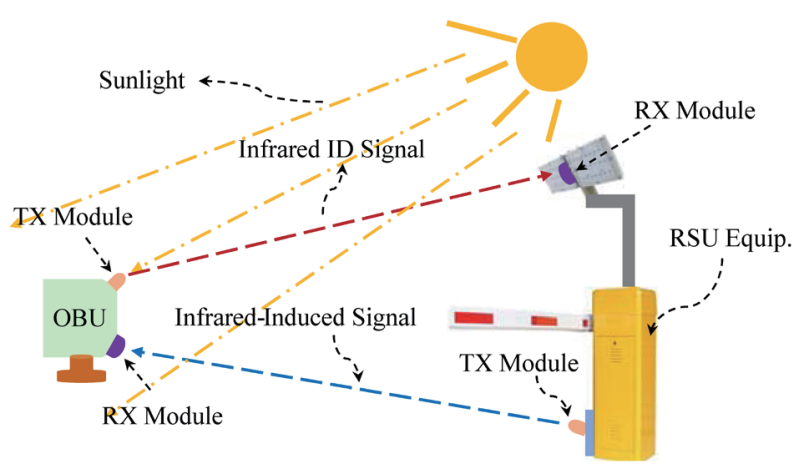

Fig. 7. (Color online) Sunlight impact reduction.

\subsection{Reduced signal interference between two neighboring vehicles}

According to Fig. 4, signal interference occurs when two neighboring vehicles enter the sensing zone at the same time. This phenomenon mainly results from the TX module of the RSU system transmitting the IR-induced signal downward, and all vehicles entering the sensing zone can receive this induced signal. In the proposed method of split TX/RX installation, because the IR-induced signal from the RSU equipment is transmitted upward, only the first vehicle entering the sensing zone receives this signal and blocks the adjacent rear vehicles from receiving the induced signal. Therefore, the signal interference caused by two neighboring vehicles in the sensing zone will be reduced as shown in Fig. 8. If the rear vehicle is much higher than the first vehicle in the sensing zone, such as in the case of a truck, the rear vehicle may receive this induced signal, but the probability of this occurrence is relatively low.

\subsection{Placement of $\mathrm{OBU}$ device in vehicle}

Unlike a conventional OBU device mounted on a car windshield, the proposed OBU device can be placed directly above the car dashboard on the right of the car. Both OBU devices basically use batteries as their power sources, and a conventional OBU device needs longer wiring from the windshield to the control panel if it requires auxiliary power. However, for the proposed OBU device, it is easier to obtain power from a USB port or cigarette lighter in the control panel. Figure 9 shows the placement positions of the OBU devices in the vehicle for the different TX/RX installation methods in the RSU system, i.e., the conventional installation and the proposed split installation.

\section{Experimental Results}

To determine the system ID rate for different methods of TX/RX installation, we built an experimental platform to simulate the operational behavior of a parking lot, where the OBU device shown in Fig. 10(a) was constructed as a simulated small car for experimental convenience. Figure 10(b) shows the RSU system prototype, which includes the main controller, the equipment with the split installation of TX and RX modules, a gate machine, a 


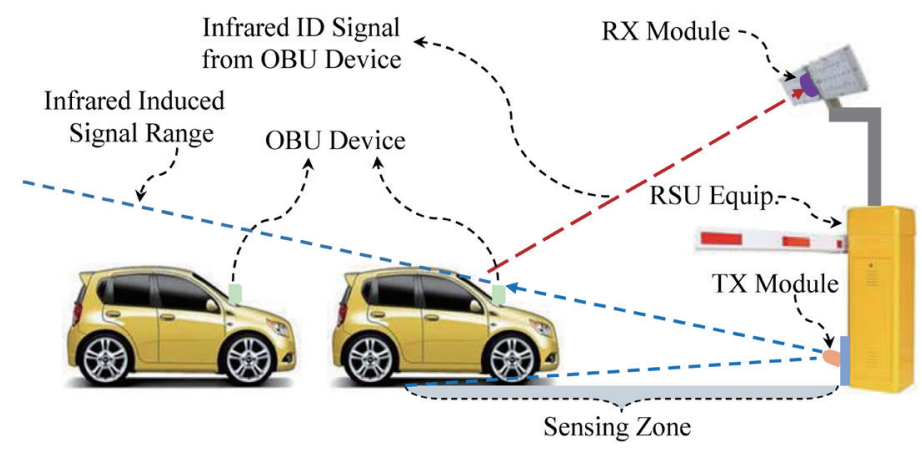

Fig. 8. (Color online) Reduced signal interference by two neighboring vehicles.

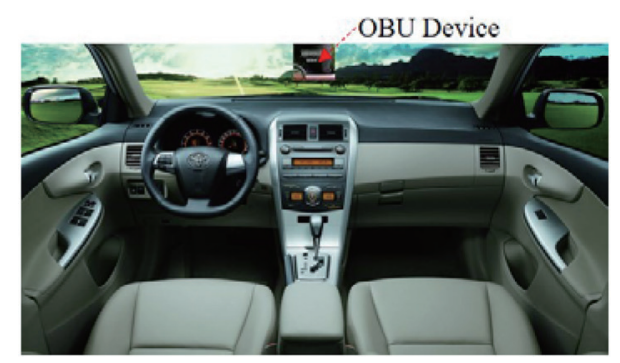

(a)

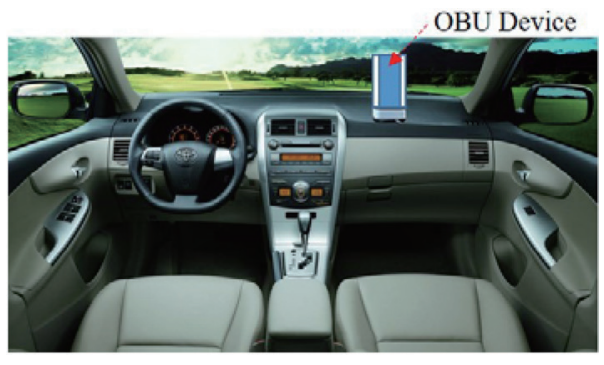

(b)

Fig. 9. (Color online) Placement positions of OBU devices for (a) conventional and (b) proposed methods.

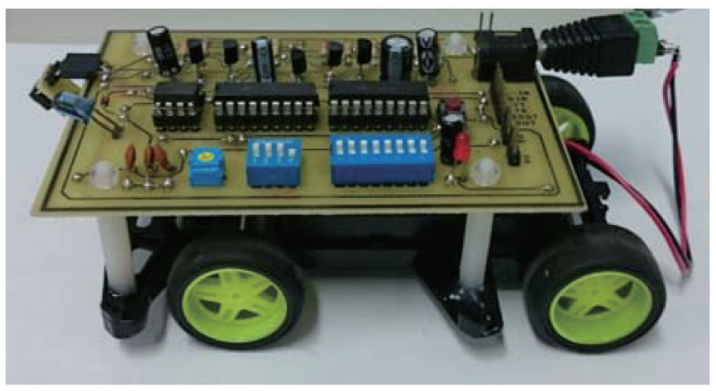

(a)

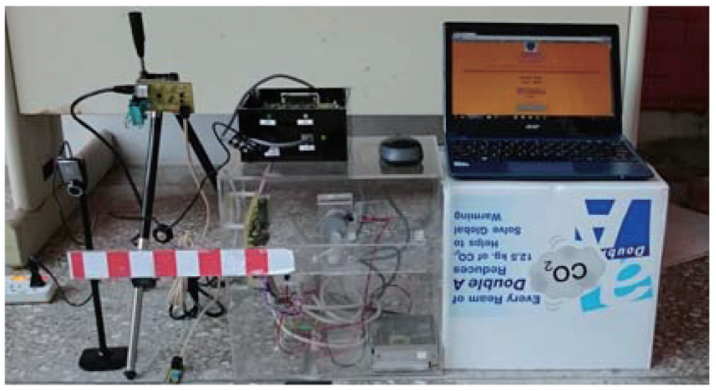

(b)

Fig. 10. (Color online) Prototypes of (a) OBU device and (b) RSU system.

CCD camera, and a backend computer. The ID code of the vehicle is determined by the data encoder (HT11E) on the OBU device, and the HT11E encoder has 8 address bits $\left(\mathrm{A}_{7} \ldots \mathrm{A}_{1} \mathrm{~A}_{0}\right)$ and 4 data bits $\left(D_{3} D_{2} D_{1} D_{0}\right)$, where 6 high address bits $\left(A_{7} \ldots A_{3} A_{2}\right)$ are used to represent the parking lot number. The remaining 2 address bits $\left(A_{1} A_{0}\right)$ plus 4 data bits $\left(D_{3} D_{2} D_{1} D_{0}\right)$ form the ID code of the vehicle, which means that the parking lot can have up to 64 parking spaces. By turning the Dual In-line Package (DIP) switches on or off, the OBU device can adjust various ID codes to represent different vehicles. The RSU system uses an embedded system module (M502) as its main controller, which has an industrial Linux-based ARM9 system on the module, which has 64 MB SDRAM, 128 MB NAND Flash, and 2 MB Data Flash. It also provides flexibility in peripheral expansion, such as Ethernet, USB 2.0, UART, GPIO, a serial SPI port, an I2C 
bus, an I2S bus, and an 8-bit local bus. ${ }^{(20)}$ Moreover, it can also support the installation of a web server and database (MySQL). This M502 controller can perform IR communication with the OBU device in the vehicle, identify the ID code of the vehicle to determine whether to open the gate, and finally connect to the backend computer to manage system information through the network. It also controls an auxiliary CCD camera via its USB port to take photos of vehicles that enter or leave the parking lot, and then transmits these photos and the corresponding information to the backend computer. Table 1 shows the components of the TX and RX modules. The main components of the TX module include a data encoder (HT12E), a modulator of the $38 \mathrm{kHz}$ carrier (NE555), and an IR transmitter constructed from several IR LEDs (TSAL6200) and an NMOS (BS170). The RX module consists of an IR receiver (IRM0208 A538) and a data decoder (HT12D).

\subsection{Comparison without interference}

In cases without any interference, such as an indoor or basement parking lot, the system ID rate for the two different installation methods mainly depends on the sensing distance. When the distance increases, the stability of the ID rate will decrease slightly because the IR signal received or transmitted by the OBU device will weaken. As shown in Fig. 11, we found that the ID rates of both installation methods of the RSU end were similar and were more than $80 \%$ even at a sensing distance of $5 \mathrm{~m}$.

Table 1

Components of TX/RX modules.

\begin{tabular}{lcllc}
\hline \multicolumn{2}{c}{ TX module } & & \multicolumn{2}{c}{ RX module } \\
\cline { 1 - 2 } \cline { 5 - 5 } Component & Part No. & & Component & Part No. \\
\cline { 1 - 2 } Encoder & HT12E & & Decoder & HT12D \\
Modulator $(38 \mathrm{kHz})$ & NE555 & & IR Receiver & IRM0208_A538 \\
IR LED & TSAL6200 & & & \\
LED Driver & BS170 & & \\
\hline
\end{tabular}

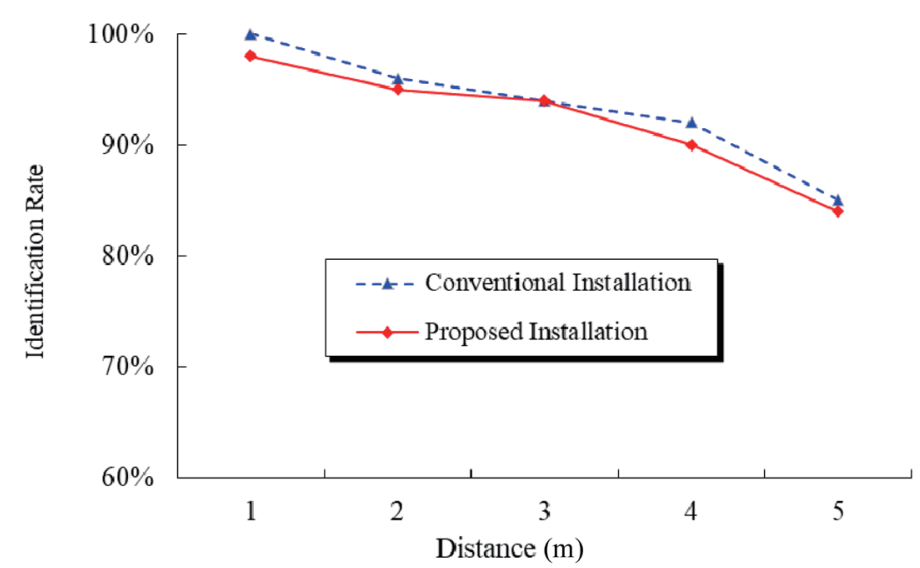

Fig. 11. (Color online) Comparison of ID rates without interference. 


\subsection{Comparison with sunlight interference}

In an experimental environment with sunlight, the ID rate of a parking lot using the conventional installation of TX/RX modules should rapidly decrease as the distance increases when sunlight directly shines on the upward receiver of the OBU device. That is because the IR content of sunlight saturates the RX module, seriously interfering with its operation, especially at a long sensing distance. However, in the proposed split TX/RX installation method on the RSU end, the RX modules of the OBU device and RSU equipment face downward to receive the IR-induced and IR ID signals, respectively. Therefore, the two RX modules can avoid direct exposure to sunlight. From Fig. 12, we indeed found that the ID rate of the RSU system using a split TX/RX installation reduced the impact of sunlight interference; even at a sensing distance of $5 \mathrm{~m}$, its ID rate was still above $70 \%$.

\subsection{Comparison of signal interference}

In our experiment, we placed two neighboring OBU devices in the sensing zone of the RSU system using the conventional TX/RX installation. As shown in Fig. 13, the ID rate immediately decreased below $50 \%$ even at a sensing distance of $1 \mathrm{~m}$ and further decreased with increasing distance. Because the two neighboring OBU devices receive the IR-induced signal from the RSU system at the same time, they almost synchronously send their ID codes back to the RSU system. These two ID codes seriously interfere with each other such that the RSU system could not make a correct ID. In the same experiment but with the proposed split TX/RX installation on the RSU system, since the RSU system transmits the IR-induced signal upward, only the OBU device of the first vehicle can receive this induced signal. For the OBU devices of the rear vehicles installed at a similar height to the OBU device of the first vehicle, the probability of receiving the induced signal will be greatly reduced. Therefore, the proposed installation method can significantly reduce the interference caused by two neighboring vehicles in the sensing zone. Figure 13 shows that the ID rate using the proposed installation method

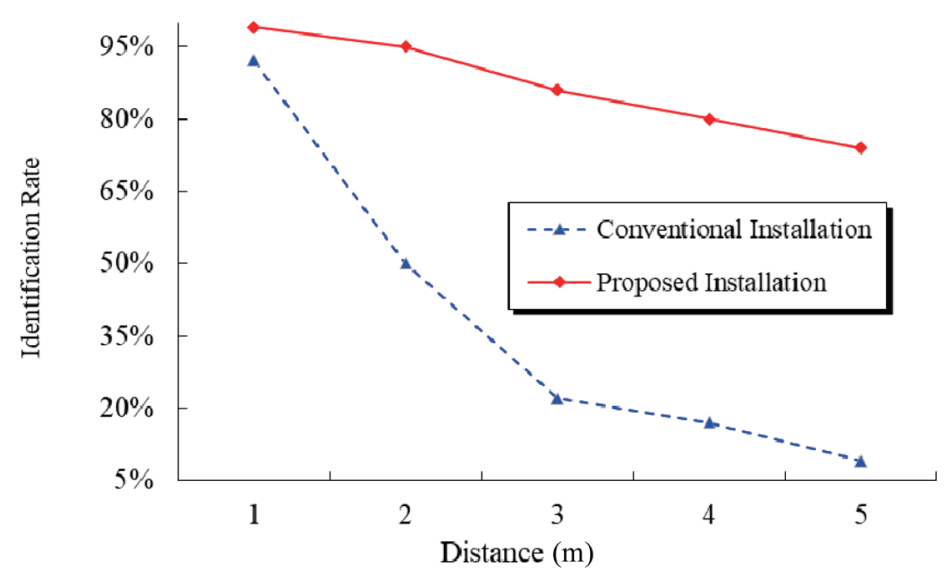

Fig. 12. (Color online) ID rate under sunlight interference. 


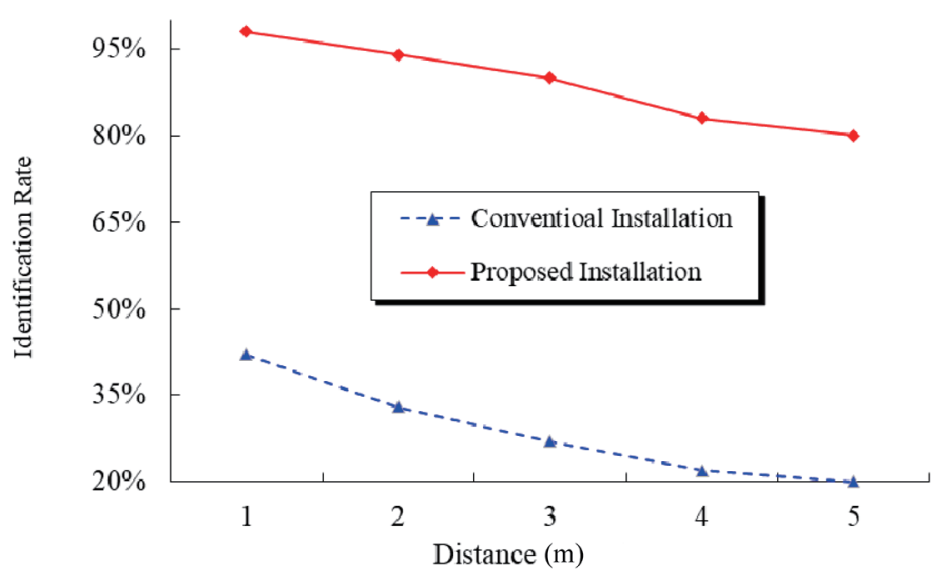

Fig. 13. (Color online) ID rate under signal interference.

remains above $70 \%$ even at a sensing distance of $5 \mathrm{~m}$, and it also demonstrates that signal interference has less effect on the proposed split TX/RX installation.

\section{Conclusions}

In this paper, a new split TX/RX installation on an RSU system is proposed for application to an IR-sensor-based parking system. According to our experimental results, the proposed installation method will effectively reduce the impact of sunlight interference and reduce the signal interference caused by two neighboring vehicles in the sensing zone. Under different types of interference, the ID rate when using the proposed installation method was above $70 \%$ in our experiment, even when the sensing distance was increased to $5 \mathrm{~m}$. Therefore, the proposed installation method indeed successfully increases the stability of system ID. The proposed system is considered to be suitable for not only indoor parking lots but also outdoor parking lots.

\section{Acknowledgments}

This work was performed under the auspices of the University of Electronic Science and Technology of China, Zhongshan Institute, China, under Grant 418YKQN11. It also received support and funding from St. John's University, Taiwan.

\section{References}

1 S. N. Myint and M. M. Oo: Am. Sci. Res. J. Eng. Technol. Sci. 26 (2016) 3. https://asrjetsjournal.org/index.php/ American_Scientific_Journal/article/view/2268

2 C. N. E. Anagnostopoulos, I. E. Anagnostopoulos, V. Loumos, and E. Kayafas: IEEE Trans. Intell. Transport. Syst. 7 (2006) 3. https://doi.org/10.1109/TITS.2006.880641

3 M. M. Rashid, A. Musa, M. A. Rahman, N. Farahana, and A. Farhana: Int. J. Mach. Learning Comput. 2 (2012) 2. https://doi.org/10.7763/IJMLC.2012.V2.95 
4 K. Masuda, S. Ogawa, Y. Takagawa, and M. Kimata: Sens. Mater. 26 (2014) 4. https://doi.org/10.18494/ SAM.2014.972

5 S. V. Herwaarden: Sens. Mater. 8 (1996) 6. https://myukk.org/SM2017/article.php?ss=10252

6 K.-C. Hu, F.-Y. Lin, C.-C. Chien, T.-S. Tsia, C.-H. Hsia, and J.-S. Chiang: 2014 IEEE Int. Conf. Consumer ElectronicsTaiwan (IEEE, 2014) 203. https://doi.org/10.1109/ICCE-TW.2014.6904058

7 C.-H. Hsia, S.-C. Yen, and J.-H. Jang: Sens. Mater. 31 (2019) 1803. https://doi.org/10.18494/SAM.2019.2351

8 J.-M. Guo, C.-H. Hsia, K. Wong, J.-Y. Wu, Y.-T. Wu, and N.-J. Wang: IEEE Trans. Veh. Technol. 65 (2016) 6. https://doi.org/10.1109/TVT.2015.2508020

9 A. Tanaka: J. Inst. Electr. Eng. Jan. 124 (2004) 12. https://doi.org/10.1541/ieejjournal.124.768

10 W. Wang, Y. Zhang, X. Kong, H. Gang, L. Zhao, and J. Lin: 2009 IEEE Int. Conf. Communications Technology and Applications (IEEE, 2009) 49. https://doi.org/10.1109/ICCOMTA.2009.5349241

11 H.-C. Chen, C.-J. Huang, and K.-H. Lu: J. Comput. 30 (2019) 1. https://doi.org/10.3966/19911599201902300101 5

12 Hamamatsu Photonics K. K.: Characteristics and Use of Infrared Detectors (2011). https://www.hamamatsu. com/resources/pdf/ssd/infrared_kird9001e.pdf

13 H.-C. Chen, C.-J. Huang, and K.-H. Lu: Proc. 2017 IEEE Int. Conf. Consumer Electronics-Taiwan (IEEE, 2017) 297. https://doi.org/10.1109/ICCE-China.2017.7991113

14 Vishay Semiconductors: https://www.vishay.com/docs/84240/vsly5940.pdf (accessed May 2019).

15 W.-Y. Shieh, C.-C. Hsu, S.-L. Tung, P.-W. Lu, T.-H. Wang, and S.-L. Chang: IEEE Trans. Intell. Transport. Syst. 12 (2011) 1. https://doi.org/10.1109/TITS.2010.2057508

16 W.-Y. Shieh, C.-C. Hsu, H.-C. Chen, T.-H. Wang, and S.-L. Chang: IET Intell. Transport. Syst. 9 (2015) 2. https://doi.org/10.1049/iet-its.2013.0096

17 M. F. Holick: Anticancer Res. 36 (2016) 3. https://www.ncbi.nlm.nih.gov/pubmed/26977036

18 J. Cai and Z. Han: Proc. 2010 IEEE/ACM Int. Conf. Green Computing and Communications \& Int. Conf. Cyber, Physical and Social Computing (IEEE, 2010) 885. https://dl.acm.org/citation. $\mathrm{cfm} ? \mathrm{id}=1953415 \& \mathrm{dl}=\mathrm{ACM} \& \mathrm{coll}=\mathrm{DL}$

19 Para Light Electronics: https://wakamatsu.co.jp/waka/PL-IRM0101-3.pdf (accessed January 2019).

20 Artila Electronics: http://www.artila.com/docs/M-502/M-502_Datasheet.pdf (accessed August 2019). 